\title{
Cerebral Artery Dissection: Spectrum of Clinical Presentations Related to Angiographic Findings
}

\author{
Jae Hyuk Kwak, MD', Jin Woo Choi, MD³, Hee Jung Park, MD², Eun Young Chae, MD', \\ Eun Suk Park, MD'1, Deok Hee Lee, MD'1, Dae Chul Suh, MD¹
}

Purpose: Cerebral arterial dissections are recognized as a common cause of stroke. However, few studies have reported on the distribution of cerebral arterial dissection and angiographic pattern related to the presenting clinical symptom pattern. We analyzed the distribution of cerebral artery dissection along with angiographic and clinical presenting a pattern as depicted on angiograms.

Materials and Methods: From January 2000 to January 2007, 133 arterial dissection patients admitted to our institutes were retrospectively reviewed. The characteristic angiographic findings of all cerebral arteries were carefully evaluated on 4-vessel angiograms. The male-female ratio was 77: 56 and the mean age was 51 years. According to the angiographic finding depicting the location of the dissection plane in the arterial wall, we categorized to steno-occlusive, aneurysmal, combined and unclassifiable pattern. In each dissection pattern, we evaluated presenting symptoms and presence of infarction or hemorrhage.

Results: The most common symptom on presentation was headache $(47 \%)$, followed by motor weakness of arm or leg (31\%), dysarthria/aphasia (19\%) and vertigo (16\%). The most common angiographic pattern was steno-occlusive (46\%), followed by combined (steno-occlusive and aneurismal) (27\%) and aneurysmal (22\%) patterns. Steno-occlusive pattern was most commonly related to infarction $(33 / 61,54 \%)$ in contrast that aneurysmal pattern was most frequently related to subarachnoid hemorrhage $(\mathrm{SAH})(7 / 29,24 \%)$. The most frequent abnormality in patients with dissection of the intradural vertebral arteries including posterior cerebral artery (PCA) was SAH $(23 / 70,33 \%)$, followed by infarction. Infarction was the most common abnormality in patients with the extradural and intradural carotid arteries, and the extradural vertebral artery.

Conclusion: In contrast that the extradural arterial dissections tended to result in ischemia with stenoocclusive pattern, the intradural arterial dissections tended to result in SAH with aneurysmal type, especially in the vertebral artery. Dissection requires combined analysis of angiographic pattern and type of stroke depending on the location.

Key Words : Cerebral arteries; Dissection; Aneurysm; Dissecting

\footnotetext{
'Department of Radiology and Research Institute of Radiology, ${ }^{2}$ Department of Internal Medicine, University of Ulsan, College of Medicine, Asan Medical Center, Seoul, Korea

${ }^{3}$ Department of Radiology, Konkuk University College of Medicine, Seoul 143-729, Korea

Received March 23, 2011; accepted after revision June 18, 2011.

Correspondence to: Dae Chul Suh, MD, Department of Radiology, University of Ulsan, College of Medicine, Asan Medical Center, 86 Asanbyeongwon-gil, Songpa-gu, Seoul 138-736, Korea.

Tel. 82.2.3010.4366 Fax. 82.2.476.0090 E-mail: dcsuh@amc.seoul.kr

This is an Open Access article distributed under the terms of the Creative Commons Attribution Non-Commercial License (http://creativecommons.org/licenses/by-nc/3.0) which permits unrestricted non-commercial use, distribution, and reproduction in any medium, provided the original work is properly cited.
} 


\section{Cerebral Artery Dissection}

Dissections are recognized as an important cause of stroke among young and middle-aged patients and account for 10 to 25 percent of these patients $[1,2]$. Head and neck pain is the most common symptom in cerebral arterial dissection. Other features vary according to location of dissection.

Dissections have been increasingly diagnosed in recent years due to development in investigative methods. A diagnosis of cerebral arterial dissection is clinically significant because it can cause severely disabling ischemic stroke or subarachnoid hemorrhage (SAH) requiring specific management according to the vessel status demonstrated on cerebral angiography. Various factors related to underlying structural defect of the arterial wall seem to play an important role in spontaneous arterial dissection [3].

Several reports have shown that the intradural vertebral artery (VA) and the cervical internal carotid artery (ICA) seem to be more frequently involved sites [4]. However, there have been few reports on the distribution of cerebral arterial dissection, angiographic pattern and relation to symptom pattern. Clinical symptomatic presentation itself does not always match to the imaging findings of dissection. In this study, we analyzed the distribution of cerebral artery dissection, angiographic pattern as depicted on angiograms in 133 patients admitted to our hospital.

\section{MATERIALS AND METHODS}

We analyzed retrospectively collected data of patients who performed cerebral angiography due to cerebrovascular symptoms in our institute from January 2000 to January 2007. The diagnosis of arterial dissection was based on the characteristic angiographic findings in association with appropriate clinical history. The angiographic examinations were performed by two neuroradiologists and/or residents under the supervision of the neuroradiologists.

To exclude atherosclerotic stenosis or dilation, the characteristic angiographic findings of atherosclerosis included vessel wall irregularity, atheromatous plaque with or without ulceration, tortuousness of all cerebral arteries were carefully evaluated on 4-vessel angiograms. One hundred thirty three patients were included in this study. The male-female ratio was 77 : 56 and the mean age was 51 years.

According to the location of dissection plane in arterial wall, dissection on angiogram was categorized to steno-occlusive or aneurysmal pattern [4-6]. The angiographic findings of the steno-occlusive pattern included a double lumen, luminal narrowing more than $30 \%$, gradual tapering ending in total occlusion of the lumen (Fig. 1A). If the ratio between the diameter of dissection and that of normal-looking distal artery is 1.5 or more, we classified to aneurysmal pattern (Fig. 1B). When the steno-occlusive pattern and aneurysmal pattern were found on angiographic analysis, we categorized this type as combined pattern (Fig. 1C). If the dissected lumen showed only flap-like filling defect, ectasia, mild narrowing less than $30 \%$, we classified these patients to unclassified pattern. The distinction of the intradural and extradural arteries was based on the observation that the ICA pierced the inner dura immediately proximal to the origin of the ophthalmic artery in the anterior circulation. Therefore, the intradural vessel was involved when a lesion was distal to the ophthalmic artery. For the VA, the distinction was made at the point where the artery pierced the dura at the level of foramen magnum. The presence of infarction or hemorrhage such as intracerebral, intraventricular or subarachnoid hemorrhage was evaluated by reviewing the CT/MRI.

\section{RESULTS}

The most common symptom on presentation was headache $(47 \%)$, followed by motor weakness of arm or leg (31\%), dysarthria/aphasia (19\%) and vertigo $(16 \%)$. Other associated symptoms included visual loss, facial palsy, neck pain, tingling sense of the arm, altered mentality and ataxia. Main presenting symptoms are listed in Table 1 according to the location of dissection.

Angiographic pattern revealed steno-occlusive $(46 \%)$, combined $(27 \%)$ and aneurysmal (22\%) patterns in the order of frequency (Table 2). Lesions were located in the anterior circulation in our 51 patients (38\%) and in the posterior circulation in 82 patients $(62 \%)$. Lesions were located intradurally in 92 patients $(69 \%)$ and extradurally in 41 patients $(31 \%)$. The most commonly involved vessels were the intradural vertebral artery (IV) (49\%) followed by the extradural carotid artery $(22 \%)$, intradural carotid artery including its branches (17\%), extradural VA $(9 \%)$ and the posterior cerebral artery (PCA) $(3 \%)$ (Table 2). In the dissection of IV, the most common angiographic pattern was combined pattern (30/66, $45 \%)$ followed by aneurysmal pattern $(21 / 66,32 \%)$.

Analysis of MR and/or CT revealed infarction (35\%), SAH (19\%), both (2\%) and none (44\%) (Table 3). Steno-occlusive pattern of cerebral artery dissection 
Jae Hyuk Kwak, et al.
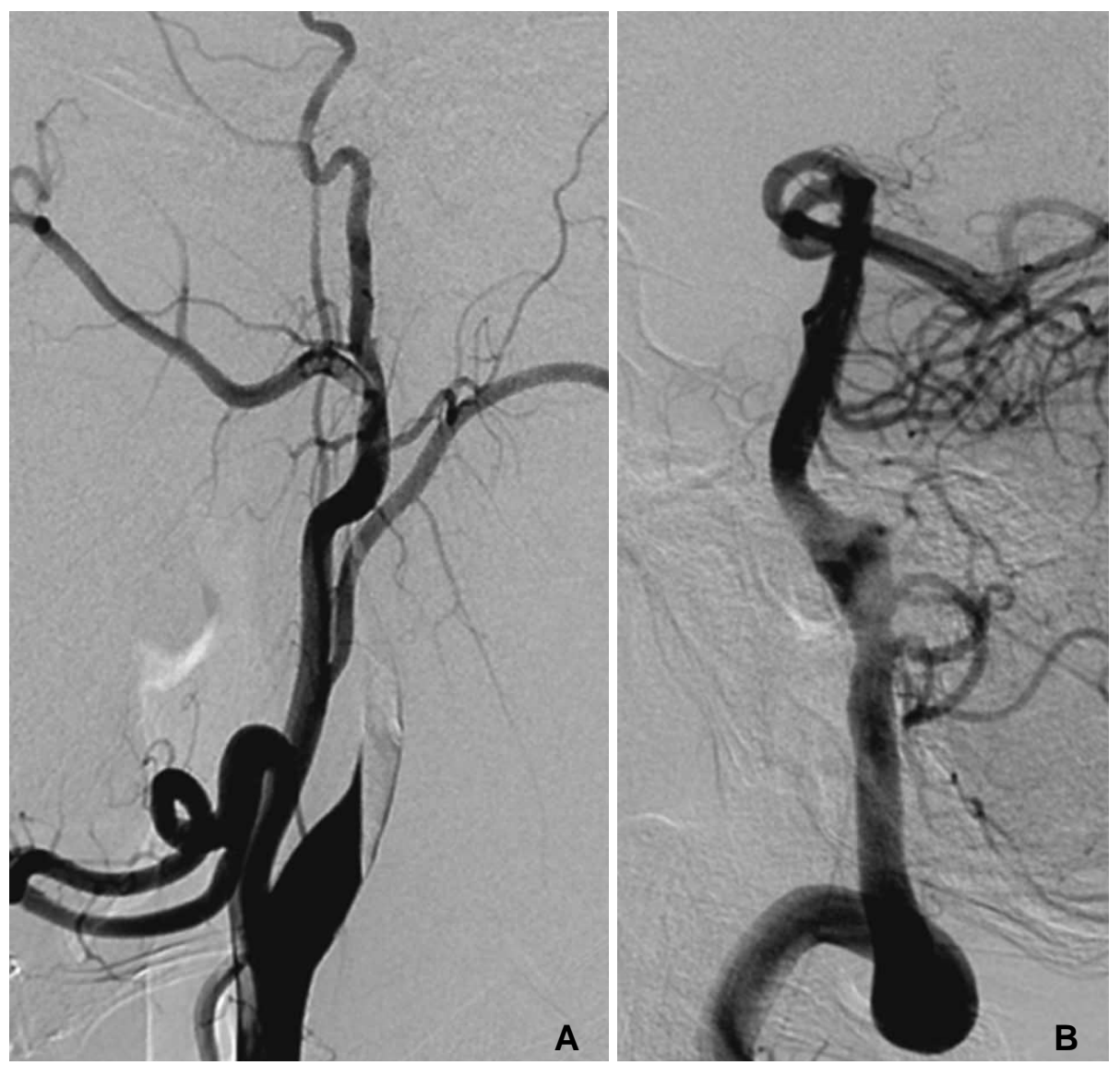

Fig. 1. A. A 46-year-old male with headache and visual loss. The right carotid angiogram shows stenoocclusive pattern of dissection involving the right cervical internal carotid artery (ICA). Several acute ischemic foci were noted in the right brain (not shown). He was clinically stable despite persistent right ICA on 5months follow-up CT angiography (not shown).

B. A 63-year-old male with subarachnoid hemorrhage (SAH). Vertebral angiogram shows fusiform aneurysmal pattern of dissection involving the vertebral artery which was subsequently embolized by coil.

C. A 50-year-old male presented with SAH. Left vertebral arteriogram shows the combined type of steno-occlusive and aneurismal pattern. Left VA including aneurismal segment was uneventfully embolized.

D. A 43-year-old female presented with initial right lateral medullary infarct and subsequent SAH. Vertebral angiogram shows fusiform aneurysmal dilatation of right vertebral artery which was embolized by coil.
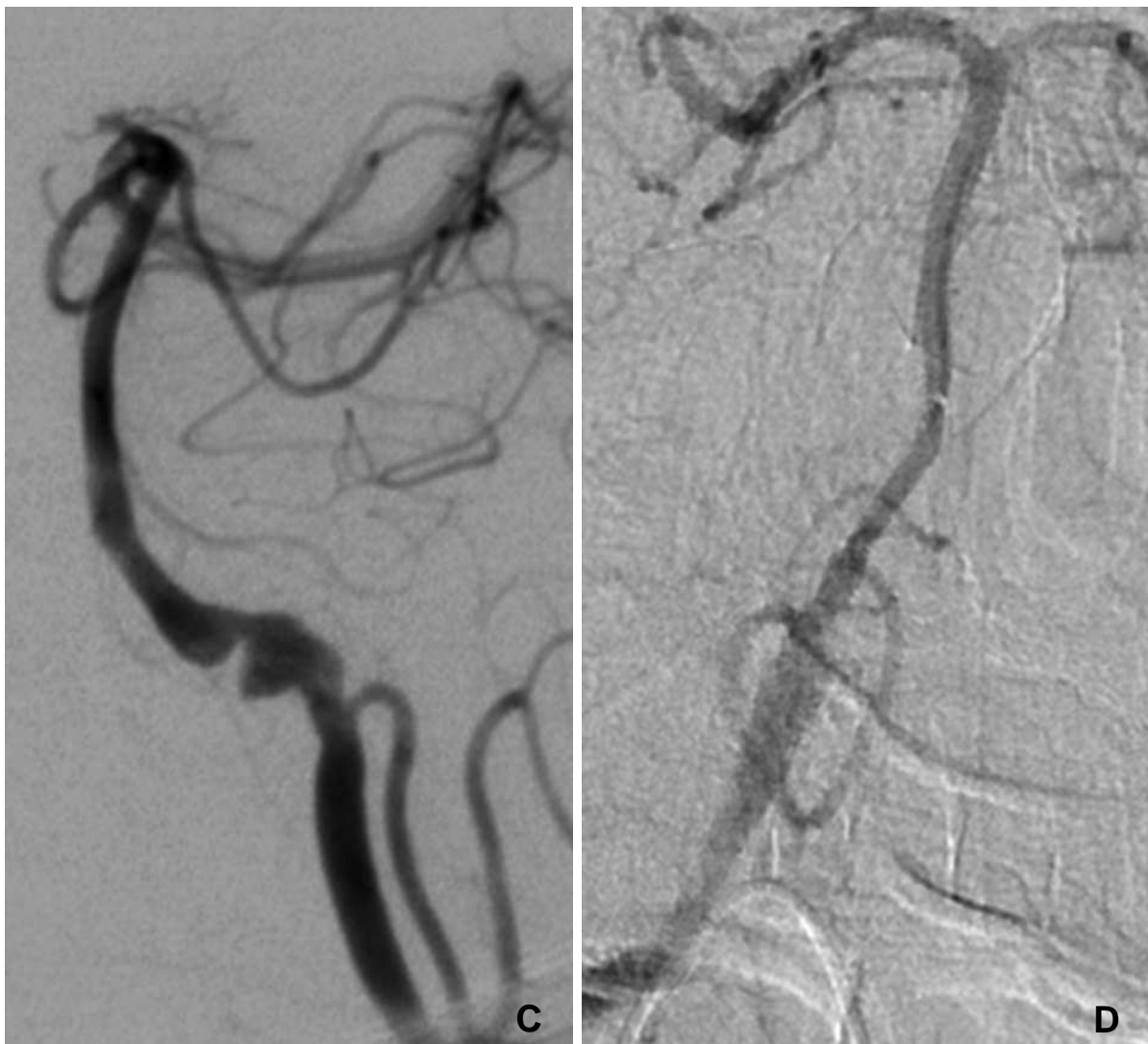


\section{Cerebral Artery Dissection}

was most commonly related to infarction $(33 / 61,54 \%)$. Aneurysmal pattern was most frequently related to SAH $(7 / 29,24 \%)$. In patients with combined pattern of both steno-occlusion and aneurysm, SAH was the most common presenting pattern on abnormal CT/MR findings (12/36, 33\%) (Table 3).

The most frequent abnormality detected on CT or MRI in patients with dissection of the IV including PCA was SAH (25/92), followed by infarction (Table 4). Infarction was the most common abnormality on CT or MRI in patients with the extradural VA, and the extradural and intradural carotid arteries. As expected, no SAH was detected in patients with dissection of extradural cerebral arteries. Three patients in intradural VA group had SAH and infarction at the same time (Fig. 1D).

\section{Rebleeding}

Among the 28 patients who presented with $\mathrm{SAH}$, we experienced 4 patients with rebleeding during the admission. Four patients had intradural vertebral arterial dissection and aneurysmal pattern. Three patients died of aggravation of their symptoms and one patient was recovered after aneurysmal clipping or extraventricular drainage.

Table 1. Presenting Symptom Related to the Location of the Dissection

\begin{tabular}{|c|c|c|c|c|c|c|}
\hline Presenting Sx & IC & IV & PCA & EC & EV & Total (\%) \\
\hline Headache & 11 & 45 & 3 & 1 & 3 & $63(47 \%)$ \\
\hline Weakness & 11 & 9 & & 19 & 3 & $42(31 \%)$ \\
\hline Aphasia / dysarthria & 7 & 3 & & 12 & 3 & $25(19 \%)$ \\
\hline Vertigo & 2 & 12 & & 2 & 6 & $22(16 \%)$ \\
\hline LOC & 1 & 3 & 2 & 1 & & $7(5 \%)$ \\
\hline Neck pain & & 4 & & 1 & & $5(4 \%)$ \\
\hline No symptom & & 3 & & 2 & & $5(4 \%)$ \\
\hline Facial palsy & 2 & & & 3 & & $5(4 \%)$ \\
\hline Visual loss & 1 & & & 2 & 1 & $4(3 \%)$ \\
\hline Ataxia & & 1 & & & & $1(1 \%)$ \\
\hline Arm tingling sense & & 1 & & & 1 & $2(1 \%)$ \\
\hline
\end{tabular}

Abbreviations: EC, extradural carotid artery; EV, extradural vertebral artery; IC, intradural carotid artery; IV, intradural vertebral artery; PCA, posterior cerebral artery; LOC, loss of consciousness; Sx, symptom

Table 2. Angiographic Patterns Related to the Location of the Dissection

\begin{tabular}{lrrrrrr}
\hline & EC & EV & IC & IV & PCA & Total \\
\hline Stenoocclusive & 19 & 9 & 18 & 14 & 1 & $31(46 \%)$ \\
Aneurysm & 2 & & 3 & 21 & 30 & $36(22 \%)$ \\
Combined & 4 & 1 & 1 & 1 & $7(5 \%)$ \\
Unclassifiable & 4 & 2 & 0 & 66 & 4 & $133(100 \%)$ \\
\hline Total & 29 & 12 & 22 & 10 & 4 \\
\hline
\end{tabular}

Abbreviations: EC, extradural carotid artery; EV, extradural vertebral artery; IC, intradural carotid artery; IV, intradural vertebral artery; PCA, posterior cerebral artery

Table 3. Clinical Findings Related to Angiographic Pattern

\begin{tabular}{lccccc}
\hline & Steno-occlusive & Aneurysm & Combined & Unclassifiable & Total \\
\hline SAH & 6 & 7 & 12 & $65(19 \%)$ & $46(35 \%)$ \\
Infarction & 33 & 2 & 6 & 1 & $3(2 \%)$ \\
Both & 22 & 2 & 17 & 2 & $59(44 \%)$ \\
None & 61 & 29 & 36 & 7 & $133(100 \%)$ \\
\hline Total & & 18 & 25 & 7 \\
\hline
\end{tabular}

Abbreviations: SAH, subarachnoid hemorrhage 
Table 4. Clinical Findings Related to the Location of Dissection

\begin{tabular}{lrrrrr}
\hline & EC & IC & EV & IV + PCA & Total \\
\hline Infarcation & 19 & 11 & 7 & 9 & 46 \\
SAH & 0 & 2 & 0 & 3 & 25 \\
Both & 0 & 0 & 0 & 5 & 35 \\
None & 10 & 9 & 12 & 70 & 59 \\
\hline Total & 29 & 22 & 5 & 73 \\
\hline
\end{tabular}

Abbreviations: SAH, subarachnoid hemorrhage; EC, extradural carotid artery; IC, intradural carotid artery; IV, intradural vertebral artery; PCA, posterior cerebral artery

\section{DISCUSSION}

Headache and neck pain was common symptoms associated with spontaneous artery dissection $[7,8]$. In our study, the most common symptom was headache $(47 \%)$, followed by motor weakness of arm or leg (31\%), dysarthria/aphasia (19\%), and vertigo (16\%). The most common presenting feature of intradural vertebral dissection was headache $(71 \%)$. However, neurologic symptoms as motor weakness and dysarthria/aphasia were more common symptom than a headache in the intradural ICA and the extradural ICA.

Prior population-based studies showed that the annual incidence of spontaneous carotid-artery dissection ranged from 2 per 100,000 to 3 per 100,000 and that of spontaneous VA dissection can be estimated at 1 per 100,000 to 1.5 per $100,000[9,10]$. In our study, the VA was more frequently involved (58\%) than the carotid artery (39\%). Intradural VA was more frequently involved than extradural VA. This result was quite different previously reported data from the western country. It might be associated with more frequent incidence of small vessel disease in Asian. In addition to a possible ethnic difference, the intracranial arterial wall differs histologically from extracranial arteries in that the media is thinner and there is no external elastic lamina [11]. It may explain the predominance of spontaneous vertebrobasilar dissection without concomitant trauma, contrasting with extracranial dissection which is more commonly post-traumatic $[7,12,13]$

The angiographic pattern related to location of arterial dissection was variable. The steno-occlusion pattern was more common in the extradural carotid and vertebral arteries, and the intradural carotid artery. The aneurysmal and combined pattern was more common in the intradural VA [14]. In contrast that stenoocclusive pattern presented with ischemic stroke, aneurysmal and combined pattern presented with SAH.
Although such dissecting aneurysm needs to be differentiated from atherosclerotic vessel wall change, underlying atherosclerotic changes might contribute to the cause of dissection [15].

There have been few reports on the cerebral artery dissection whose distribution was analyzed in the concept of diameter of the vessels. We supposed that the diameter of cerebral artery contributed to decide the type of dissection. A subintimal dissection tends to result in stenosis of the arterial lumen that may cause infarction due to flow disturbance. On the other hand, a subadventitial dissection may cause aneurysmal dilation of the artery, which can cause SAH $[16,17]$. Ohkuma et al. reported that arterial stenosis with dilatation occurred predominantly in SAH cases, whereas stenosis without dilatation arose mainly in ischemic cases $[17,18]$. Because the ICA and the extradural VA have the larger diameter than the intradural VA and PCA, they have thicker media which tends to maintain the adventitia of carotid artery. The intradural carotid artery, the extradural carotid artery and the extradural VA group presented frequently with ischemia as in our study because they might have more subintimal than subadventitial type dissections. However, in the anterior circulation, dissection was more frequently involved in the extradural ICA group (22\%) compared to the intradural ICA group (17\%) despite of the larger diameter of the extradural than the intradural carotid arteries.

Our study revealed that dissection of the intradural VA was the most common site and was the most common cause of SAH. The main trunk of VA has a long part in the subarachnoid space and the intradural segment of the VA has a thinner media and adventitia, and dissections involving the segment are more likely to extend subadventitially producing SAH. This may explain that rebleeding was associated with the intradural vertebral arterial dissection as in four of our patients even though the incidence is not high [19].

Dissection has variable clinical outcome depending 


\section{Cerebral Artery Dissection}

on location and angiographic pattern. Dissection of the extradural arteries results in more occurrences of ischemia caused by steno-occlusive pattern. Intradural arterial dissections result in more occurrence of $\mathrm{SAH}$ caused by aneurysmal pattern, especially in the vertebral artery. The most frequent abnormality in patients with dissection of the intradural vertebral arteries was SAH and followed by infarction. Careful analysis and follow-up based on the angiographic finding and clinical presentation is mandatory because some patients presented with SAH in steno-occlusive pattern or infarction in aneurismal pattern.

\section{References}

1. Ducrocq X, Lacour JC, Debouverie M, Bracard S, Girard F, Weber M. Accidents vasculaares cérébraux ischémiques du sujet jeune. Étude prospective de 296 patients âgés de 16 à 45 ans. Rev Neurol 1999; 155:575-582

2. Hart RG, Easton JD. Dissections. Stroke 1985;16:925-927

3. Krings T, Choi IS. The many faces of intracranial arterial dissections. Interv Neuroradiol 2010;16:151-160

4. Shin JH, Suh DC, Choi CG, Leei HK. Vertebral artery dissection: spectrum of imaging findings with emphasis on angiography and correlation with clinical presentation. Radiographics 2000;20: 1687-1696

5. Hart RG, Easton JD. Dissections of cervical and cerebral arteries Neurol Clin 1983;1:155-182

6. Touzé E, Randoux B, Méary E, Arquizan C, Meder JF, Mas JL. Aneurysmal forms of cervical artery dissection : associated factors and outcome. Stroke 2001;32:418-423

7. Li S, Yan B, Kaye A, Mitchell P, Dowling R, Collins M, et al. Prognosis of intracranial dissection relates to site and presenting features. J Clin Neurosci 2011;18:789-793

8. Silbert PL, Mokri B, Schievink WI. Headache and neck pain in spontaneous internal carotid and vertebral artery dissections. Neurology 1995;45:1517-1522

9. Bogousslavsky J, Regli F. Ischemic stroke in adults younger than 30 years of age. Cause and prognosis. Arch Neurol 1987;44:479482

10. Lucas C, Moulin T, Deplanque D, Tatu L, Chavot D. Stroke patterns of internal carotid artery dissection in 40 patients. Stroke 1998;29:2646-2648

11. Strong KC. A study of the structure of the media of the distributing arteries by the method of microdissection. The Anatomical Record 1938;72:151-167

12. Kocaeli H, Chaalala C, Andaluz N, Zuccarello M. Spontaneous intradural vertebral artery dissection: a single-center experience and review of the literature. Skull Base 2009;19:209-218

13. Jang YG, Ryu CW, Kim JS, Cha EY, Pyun HW, Kim DY, et al. Dissecting aneurysm of the basilar arterial trunk presenting with pontine infarction: coil obliteration of the dissecting aneurysm including the diseased basilar arterial segment. Interv Neuroradiol 2007;13:381-384

14. Mizutani T, Miki Y, Kojima H, Suzuki H. Proposed classification of nonatherosclerotic cerebral fusiform and dissecting aneurysms. Neurosurgery 1999;45:253-259

15. In HS, Lee HY, Park JY, Kim SY, Jung JH, Kim JS, et al. Intracranial stenting in patients with atherosclerotic stenosis associated with various aneurysms in the same diseased arterial segment. AJNR Am J Neuroradiol 2010;31:1895-1898

16. Yonas H, Agamanolis D, Takaoka Y, White RJ. Dissecting intracranial aneurysms. Surg Neurol 1977;8:407-415

17. Yamada SM, Nakane M, Aoki M, Nakayama H. Dissecting aneurysm of the anterior cerebral artery with severe subarachnoid hemorrhage during treatment for cerebral infarction. Intern Med 2003;42:433-435

18. Ohkuma H, Suzuki S, Ogane K. Dissecting aneurysms of intracranial carotid circulation. Stroke 2002;33:941-947

19. Mizutani T. Natural course of intracranial arterial dissections. $J$ Neurosurg 2011;114:1037-1044 